\title{
Innovación docente en la asignatura de Ética y Deontología Profesional del Grado en Psicología
}

\section{University Teaching Innovation in the subject of Professional Ethics and Deontology in the Degree in Psychology}

JEsÚS FUnUYET-SALAS

https://orcid.org/0000-0002-4133-140X

Universidad de Sevilla

Departamento de Personalidad, Evaluación y Tratamiento Psicológicos

jfunuyet1@us.es

DOI: http://dx.doi.org/10.12795/9788447231003.020

Pp.: 423-445 


\section{Descripción del contexto de la asignatura}

El presente ciclo de mejora (CIMA) ha sido aplicado en la asignatura de Ética y Deontología Profesional del Grado en Psicología de la Universidad de Sevilla, durante el primer cuatrimestre del curso 2020/2021. Se trata de la única asignatura obligatoria del cuarto curso del Grado, con 6 créditos que impartimos cuatro profesores del Departamento de Personalidad, Evaluación y Tratamiento Psicológicos. Mi docencia se corresponde con los créditos prácticos de la asignatura, llevándose a cabo de manera completamente presencial. Mi grupo ha estado constituido por 17 estudiantes, la mayoría de edades comprendidas entre los 21 y los 25 años, distribuidos en el aula manteniendo una distancia de seguridad como consecuencia de la situación de emergencia sanitaria.

Los créditos prácticos de la asignatura tradicionalmente se han organizado en torno a dos grandes bloques temáticos: por una parte, se trabaja con informes periciales y, por otra parte, el abordaje de casos que implican un dilema ético para el profesional de la psicología, situándose el CIMA en este segundo bloque de contenidos. Se pretende que los estudiantes, tras su paso por la asignatura, hayan integrado una serie de conocimientos y valores relativos a los principios y normas éticas en el ejercicio de la psicología, que les permitan afrontar y resolver ética y adecuadamente futuros dilemas y conflictos que se les puedan presentar durante su vida laboral.

\section{Diseño previo del CIMA}

\section{Mapa de contenidos y problemas}

El CIMA ha sido diseñado alrededor de seis problemas clave que nos interpelan sobre el procedimiento a seguir a la hora de analizar y resolver distintas situaciones que implican dilemas éticos en el ejercicio de la psicología. Como se puede observar en la Figura 1, cada uno de

Ciclos de Mejora en el Aula (2020). Experiencias de Innovación Docente de la US Esta obra se distribuye con la licencia Creative Commons 
estos problemas clave emana de un contenido conceptual. Así, dando respuesta a la pregunta “Cómo podemos resolver conflictos éticos sin romper la confidencialidad del cliente?" se estará abordando el principio de confidencialidad, es decir, se analizarán los pasos a seguir para dar respuesta a dilemas éticos sin vulnerar el principio de confidencialidad. En contraposición, ante la cuestión “Cómo deberíamos actuar en situaciones en las que la vida o derechos fundamentales de alguien está en juego?", se trabajará la respuesta ante situaciones en las que tenemos el deber de evitar un daño al paciente o a una tercera persona. Otro problema clave a trabajar en clase será “Cómo resolvemos situaciones en las que los progenitores están superando los límites de la patria potestad sobre sus hijos?, a raíz del cual los alumnos aprenderán a resolver situaciones en las que se está produciendo un abuso de la patria potestad por parte de los progenitores o tutores legales de un menor. Asimismo, mediante la pregunta “Cómo deberíamos actuar si nos vemos involucrados en una intervención psicoterapéutica fraudulenta?", los alumnos aprenderán cómo se debe actuar ante el conocimiento o implicación directa en una situación que supone una pseudoterapia o intervención psicoterapéutica sin fundamento científico. También se abordará en clase la cuestión “Cómo podemos resolver conflictos éticos comunes en el ámbito laboral de la psicoterapia?", con la que se pretende que los estudiantes sepan cómo resolver situaciones éticas problemáticas en las que se pueden ver inmersos en su futuro laboral. Y, por último, se trabajarán las relaciones duales a partir de la pregunta “Qué pasos habría que seguir para resolver conflictos éticos derivados de una relación dual?".

Todos estos problemas clave serán abordados siguiendo un modelo de toma de decisiones (Canadian Psychological Association, 2017), cuyos pasos vienen recogidos en la Figura 1, no obstante, se resumen en tres: identificar la situación ética problemática (el dilema); desarrollar distintas alternativos o cursos de acción, analizando sus

Ciclos de Mejora en el Aula (2020). Experiencias de Innovación Docente de la US Esta obra se distribuye con la licencia Creative Commons 
posibles riesgos y beneficios; y elegir y justificar el mejor curso de acción para resolver el dilema.

Mediante el aprendizaje de los conceptos descritos se pretende, finalmente, fomentar en los alumnos la reflexión sobre los valores personales y culturales, y el desarrollo de actitudes morales consecuentes con los valores adoptados, así como una actitud de respeto e implicación con las normas éticas que tienen que ver con el ejercicio profesional de la psicología. Asimismo, desarrollar una actitud de respeto hacia la pluralidad metodológica de la psicología, siempre que se encuentre dentro de los límites del conocimiento científico, y recalcar la importancia de la negociación a la hora de resolver dilemas éticos.

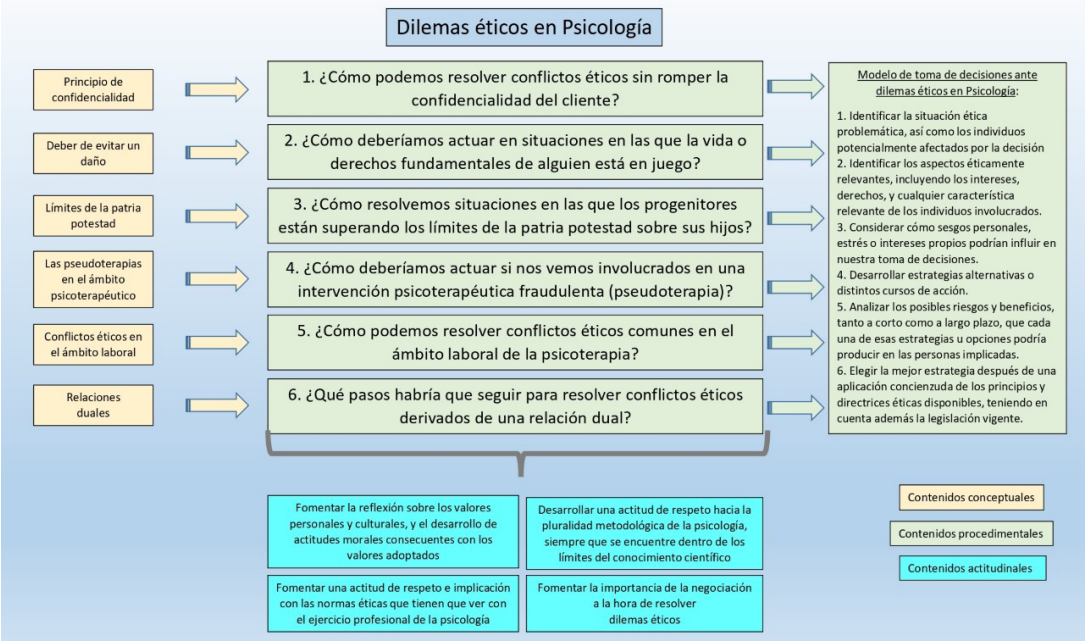

Figura 1. Mapa de contenidos y problemas

Modelo metodológico posible y secuencia de actividades programadas

Cada uno de los seis problemas clave descritos anteriormente será abordado en base al siguiente modelo metodológico (Figura 2): se partirá de un repaso de la sesión anterior que sirva de introducción de la sesión, para pasa a plantear el problema o dilema a resolver. Los alumnos, organizados en grupos de cuatro o cinco integrantes, 
debatirán con sus compañeros de grupo sobre la posible resolución del caso. A continuación, el profesor pasará a debatir exclusivamente sobre el problema con uno de los grupos, que previamente se habrá presentado como voluntario, proporcionándole a su vez una serie de consignas que le permitirán, posteriormente, ejercer como moderador de un debate con el resto de grupos de la sesión. El objetivo de este debate entre el grupo moderador y el resto de los grupos será llegar, entre todos, a la mejor resolución del dilema. Una vez concluido el debate, se terminará apuntando las conclusiones del caso y abriendo un turno de dudas y preguntas.

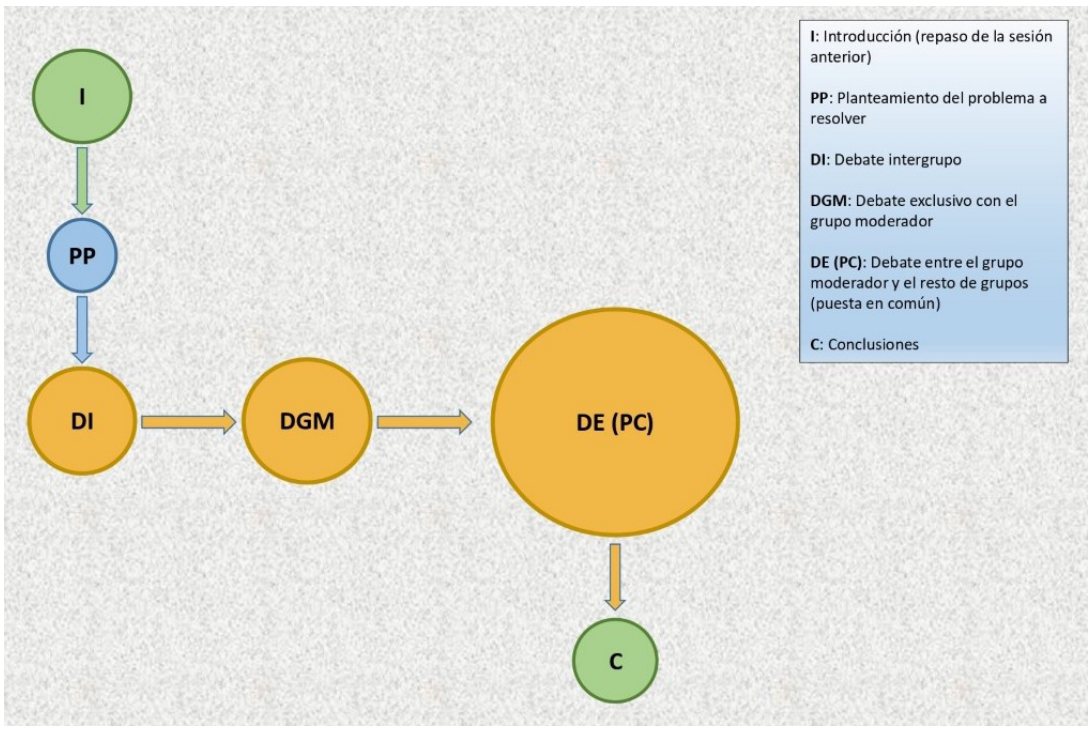

Figura 2. Modelo metodológico posible

Este modelo metodológico será aplicado a lo largo de cuatro sesiones, de dos horas cada una. La secuencia de actividades propuesta para la primera sesión (Tabla 1) abordará el primero de los problemas clave, la resolución de conflictos éticos sin romper la confidencialidad del cliente, mediante el análisis y debate de dos casos prácticos. El primero de ellos, que se trabajará durante la primera mitad de la sesión, implicará analizar un dilema en

Ciclos de Mejora en el Aula (2020). Experiencias de Innovación Docente de la US Esta obra se distribuye con la licencia Creative Commons 
el cual un adolescente de dieciséis años confiesa al psicólogo del instituto que tiene un grave problema de adicción a las drogas, que está afectando a todas las áreas de su vida, pero nos exige no contar nada a sus padres. Este caso será analizado y resuelto partiendo de las ideas previas de los alumnos, sin directrices previas, pasando a explicar posteriormente el modelo de toma de decisiones ante dilemas éticos en psicología (Figura 1), a partir del cual se trabajará el segundo caso de la sesión (una adolescente de diecisiete años que confiesa al psicólogo del instituto que tiene un retraso en la menstruación y que cree estar embarazada, y que quiere abortar como sea, pero nos prohíbe contar nada a sus padres), así como el resto de dilemas que componen el CIMA.

La segunda sesión será dedicada a dar respuesta, de nuevo a raíz de dos casos prácticos, al segundo problema clave: cómo actuar en situaciones en las que la vida o derechos fundamentales de una persona está en juego. Durante la primera mitad de la sesión se trabajará el primer caso: tras años en situación de desempleo, somos contratados en una institución privada gracias una psicóloga que trabaja allí, que es amiga nuestra desde hace tiempo. Tras un tiempo trabajando en la institución, nos enteramos de que esta psicóloga maltrata a algunos pacientes internos, ante lo que nos surge un dilema ético sobre cómo proceder. Tras su análisis y debate, en la segunda mitad de la sesión se abordará un caso en el cual una paciente a la que llevamos tratando durante unos meses por un trastorno de depresión mayor como consecuencia del fallecimiento de su hija, nos indica en consulta su inminente intención de suicidarse por la llegada del aniversario de la muerte de su hija.

Durante la primera mitad de la tercera sesión se trabajará el tercero de los problemas clave, cómo resolver situaciones en las que los progenitores están superando los límites de la patria potestad sobre sus hijos. Para ello, se analizará y debatirá un dilema en el cual un equipo de

Ciclos de Mejora en el Aula (2020). Experiencias de Innovación Docente de la US Esta obra se distribuye con la licencia Creative Commons 
profesores en un colegio solicita la evaluación e intervención por parte de un logopeda de un niño de diez años con graves problemas en el lenguaje, que afectan a su desarrollo personal y académico. Sin embargo, los padres del niño se niegan, a pesar de que se les informa de los perjuicios para su hijo que tendría no hacerlo. La segunda mitad de la sesión se dedicará a dar respuesta al cuarto problema clave del CIMA: cómo actuar si nos vemos involucrados en una intervención psicoterapéutica fraudulenta. Se trabajará un caso en el que un psicólogo recién egresado consigue su primer trabajo en una clínica que se publicita informando de la aplicación de intervenciones cognitivo-conductuales. Sin embargo, la directora de la clínica en realidad trata a sus pacientes desde pseudoterapias como el reiki o las flores de Bach, y le dice al psicólogo que estas son las técnicas que realmente funcionan, y que le enseñará a usarlas.

Por último, la cuarta sesión se destinará a dar respuesta a los dos últimos problemas clave. Primero, cómo resolver conflictos éticos comunes en el ámbito laboral de la psicoterapia, para lo que se trabajará el siguiente caso: la directora de un gabinete de psicología contrata a una psicóloga para que intervenga sobre los casos que le sean derivados, cobrando el $50 \%$ de los honorarios que paga el cliente. Sin embargo, con el paso de los meses y con la llegada de la crisis económica, la directora comienza a derivarle a la psicóloga fundamentalmente pacientes que proceden de una mutua, que solo pagan el $25 \%$ de la tarifa establecida. Se genera un conflicto ante la intención de la psicóloga de abandonar el gabinete para fundar por su cuenta una nueva clínica, pues cuando firmó el contrato aceptó que los pacientes pertenecían al gabinete, y por lo tanto debería continuar alli el tratamiento hasta darles el alta. Posteriormente, será abordado el sexto problema clave: qué pasos habría que seguir para resolver conflictos éticos derivados de una relación dual. Se analizará y debatirá un dilema surgido tras aceptar como paciente a la mujer de un antiguo paciente que falleció hace dos años como 
consecuencia de una enfermedad incurable. Pasadas unas sesiones, la mujer comienza a hacer preguntas sobre su marido, sobre lo que pensaba de ella o de la relación que mantenían, lo cual sitúa al terapeuta en una situación incómoda y que obstaculiza la relación terapéutica.

Tabla 1. Ejemplo de secuencia de actividades

\begin{tabular}{|c|c|c|}
\hline \multicolumn{2}{|c|}{$\begin{array}{c}\text { Sesión 1. Primera parte: ¿Cómo podemos resolver conflictos } \\
\text { éticos sin romper la confidencialidad del cliente? }\end{array}$} & \multirow{2}{*}{$\begin{array}{l}60 \\
\text { minutos } \\
10 \\
\text { minutos }\end{array}$} \\
\hline 1 & Introducción: ¿Qué implica un dilema ético? & \\
\hline \multicolumn{3}{|c|}{$\begin{array}{l}\text { Descripción: } \\
\text { Se inicia el bloque temático introduciendo la noción de "dilema ético", } \\
\text { preguntando al alumnado sobre sus ideas previas acerca del significado y lo } \\
\text { que implica un dilema ético en el contexto de la psicología. A continuación, } \\
\text { se forman los grupos de trabajo, que estarán constituidos por cuatro o } \\
\text { personas. Se mantendrán los mismos grupos de trabajo a lo largo de las } \\
\text { cuatro sesiones del CIMA. }\end{array}$} \\
\hline 2 & Planteamiento del problema & $\begin{array}{c}5 \\
\text { minutos } \\
\end{array}$ \\
\hline \multicolumn{3}{|c|}{$\begin{array}{l}\text { Descripción: } \\
\text { Se pide que uno de los grupos de trabajo se presente como voluntario para } \\
\text { la tarea que vamos a realizar, sin decir de qué se trata. Una vez tenemos el } \\
\text { grupo voluntario, se les entrega un folio donde viene planteado el problema } \\
\text { a resolver. El grupo voluntario presentará al resto de grupos el caso, que } \\
\text { en resumen se trata de: un adolescente de dieciséis años nos confiesa a } \\
\text { nosotros, como psicólogo del instituto, que tiene un grave problema de } \\
\text { adicción a las drogas, que está afectando a todas las áreas de su vida, pero } \\
\text { nos exige no contar nada a sus padres. }\end{array}$} \\
\hline 3 & Debate intergrupo & $\begin{array}{l}10 \\
\text { minutos }\end{array}$ \\
\hline \multicolumn{3}{|c|}{$\begin{array}{l}\text { Descripción: } \\
\text { Cada grupo piensa y debate, a nivel interno (no en común), como resolvería } \\
\text { el caso, sin más directrices al respecto. Se pretende conocer cuáles son las } \\
\text { ideas previas que tiene el alumnado sobre cómo deberíamos resolver un } \\
\text { caso que supone un dilema ético para el profesional de la psicología. }\end{array}$} \\
\hline 4 & Debate exclusivo con el grupo moderador & $\begin{array}{c}10 \\
\text { minutos }\end{array}$ \\
\hline
\end{tabular}

Ciclos de Mejora en el Aula (2020). Experiencias de Innovación Docente de la US Esta obra se distribuye con la licencia Creative Commons Reconocimiento-NoComercial-SinObraDerivada 4.0 Internacional (CC BY-NC-ND 4.0.) 
Descripción:

Me acerco al grupo voluntario, que ejercerá a continuación como grupo moderador. El resto de grupos, mientras, estarán preparando por escrito su opinión sobre la mejor resolución para el caso, para enviármela por email. Debato con el grupo moderador sobre cómo resolverían el caso. En el caso de que se alejen de la resolución recomendada, les orientaré mediante preguntas, teniendo en cuenta la dificultad que supone resolver un dilema ético por primera vez, y sin directrices. Una vez lleguemos a un consenso, les pediré que a continuación moderen el debate con el resto de grupos. Les daré indicaciones acerca de dejar que los compañeros piensen, no dándoles la resolución directamente. También que les hagan cuestionarse sobre aquellas opciones de resolución no recomendadas por ser poco éticas, para que los demás grupos argumenten su postura ante tales opciones. No obstante, los tranquilizaré ofreciéndoles mi disponibilidad para ayudarles a moderar el debate, si en algún momento no saben cómo continuar.

\begin{tabular}{|c|c|c|}
\hline 5 & $\begin{array}{c}\text { Debate entre el grupo moderador y el resto de grupos } \\
\text { (puesta en común) }\end{array}$ & $\begin{array}{c}20 \\
\text { minutos }\end{array}$ \\
\hline \multicolumn{3}{|c|}{$\begin{array}{l}\text { Descripción: } \\
\text { El grupo moderador pide al resto de grupos que expongan sus argumentos } \\
\text { para solucionar el caso. Cada grupo hablará en voz de un portavoz previamente } \\
\text { acordado, y se desarrollará el debate. Yo como profesor intervendré en el } \\
\text { caso de que vea que es necesario, llevando la batuta del debate el grupo } \\
\text { moderador. Entre todos se pretende llegar a la opción de resolución idónea, } \\
\text { justificándola y argumentando los motivos que hacen descartar el resto de } \\
\text { opciones surgidas en el debate. }\end{array}$} \\
\hline
\end{tabular}

6

Conclusiones

5

minutos

Descripción:

Expondré los puntos clave de resolución del caso, para reforzar las conclusiones extraídas del debate.

\begin{tabular}{|c|c|c|}
\hline \multicolumn{2}{|c|}{$\begin{array}{c}\text { Sesión 1. Segunda parte: ¿Cómo podemos resolver conflictos } \\
\text { éticos sin romper la confidencialidad del cliente? }\end{array}$} & $\begin{array}{c}60 \\
\text { minutos }\end{array}$ \\
\hline 1 & $\begin{array}{c}\text { Introducción: Modelo de toma de decisiones ante dilemas } \\
\text { éticos en psicología }\end{array}$ & $\begin{array}{c}10 \\
\text { minutos }\end{array}$ \\
\hline $\begin{array}{l}\text { Descripción: } \\
\text { Se comentará que en el caso visto durante la primera parte de la sesión, cada } \\
\text { grupo ha abordado el caso de la mejor forma que ha sabido, sin directrices } \\
\text { previas, pero que existe un modelo de toma de decisiones ante situaciones } \\
\text { que implican un dilema ético en psicología (Figura 1). Se propondrá seguir } \\
\text { este modelo de toma de decisiones a partir de ahora a la hora de resolver } \\
\text { los dilemas. }\end{array}$ \\
\hline
\end{tabular}

Ciclos de Mejora en el Aula (2020). Experiencias de Innovación Docente de la US Esta obra se distribuye con la licencia Creative Commons Reconocimiento-NoComercial-SinObraDerivada Internacional (CC BY-NC-ND 4.0.) 
Descripción:

Se pide que uno de los grupos de trabajo se presente como voluntario para ejercer de grupo moderador para el siguiente caso (sin contar al grupo que ya ejerció de moderador en la primera parte de la sesión). Una vez tenemos el grupo moderador, se les entrega un folio donde viene planteado el problema a resolver. El grupo moderador presentará al resto de grupos el caso, que en resumen se trata de: una adolescente de diecisiete años nos confiesa a nosotros, como psicólogo del instituto, que tiene un retraso en la menstruación y que cree estar embarazada, ya que mantiene relaciones sexuales de riesgo. Tiene previsto abortar como sea, sin embargo, sus padres no podrían enterarse bajo ningún concepto, o la echarían de casa y toda su vida se desmoronaría.

3 Debate intergrupo

Descripción:

Cada grupo piensa y debate, a nivel interno (no en común), como resolvería el caso, basándose en el modelo de toma de decisiones.

\begin{tabular}{|c|c|c|}
\hline 4 & Debate exclusivo con el grupo moderador & 10 \\
minutos
\end{tabular}

Me acerco al grupo moderador para debatir con ellos cómo resolverían el caso. El resto de grupos, mientras, estarán preparando por escrito su opinión sobre la mejor resolución para el caso, para enviármela por email.

En el debate con el grupo moderador, les pido su opinión sobre el caso, y si se alejan de la resolución recomendada, les orientaré mediante preguntas. Una vez lleguemos a un consenso, les pediré que a continuación moderen el debate con el resto de grupos. Les daré indicaciones acerca de dejar que los compañeros piensen, no dándoles la resolución directamente. También que les hagan cuestionarse sobre aquellas opciones de resolución no recomendadas por ser poco éticas, para que los demás grupos argumenten su postura ante tales opciones. No obstante, los tranquilizaré ofreciéndoles mi disponibilidad para ayudarles a moderar el debate, si en algún momento no saben cómo continuar.

\begin{tabular}{|c|c|c|}
\hline 5 & $\begin{array}{c}\text { Debate entre el grupo moderador y el resto de grupos } \\
\text { (puesta en común) }\end{array}$ & $\begin{array}{c}20 \\
\text { minutos }\end{array}$ \\
\hline
\end{tabular}

Ciclos de Mejora en el Aula (2020). Experiencias de Innovación Docente de la US Esta obra se distribuye con la licencia Creative Commons Reconocimiento-NoComercial-SinObraDerivada Internacional (CC BY-NC-ND 4.0.) 
Descripción:

El grupo moderador pide al resto de grupos que expongan sus argumentos para solucionar el caso. Cada grupo hablará en voz de un portavoz previamente acordado, desarrollándose el debate. Yo como profesor intervendré en el caso de que vea que es necesario, llevando la batuta del debate el grupo moderador. Entre todos se pretende llegar a la opción de resolución idónea, justificándola y argumentando los motivos que hacen descartar el resto de opciones surgidas en el debate.

6

Conclusiones

5

minutos

Descripción:

Expondré los puntos clave de resolución del caso, para reforzar las conclusiones extraídas del debate, asi como las conclusiones que podemos extraer acerca de la confidencialidad en dilemas éticos. Clausura de la sesión.

\section{Cuestionario inicial-final}

Para conocer las ideas previas de los alumnos y valorar su aprendizaje posterior, elaboré un cuestionario (Figura 3) que fue administrado antes de iniciar el CIMA y tras su finalización. Cada una de las preguntas del cuestionario están dedicadas a conocer las ideas de los alumnos sobre cada uno de los problemas claves del CIMA. Así, la primera pregunta se centra en el principio de confidencialidad. La segunda pregunta aborda una situación en la que se debe evitar un daño a una tercera persona. La tercera cuestión trata sobre los límites del uso de la patria potestad. La cuarta pregunta está enfocada en torno a un conflicto ético en el ámbito laboral. La quinta cuestión aborda las relaciones duales. Por último, la sexta pregunta se centra en la resolución de conflictos relacionados con intervenciones psicoterapéuticas fraudulentas.

Ciclos de Mejora en el Aula (2020). Experiencias de Innovación Docente de la US Esta obra se distribuye con la licencia Creative Commons 
Imagina que recientemente has sido contratado para formar parte de la comisión deontológica del Colegio de Psicologia, y para empezar te han llegado una serie de consultas de colegas solicitando asesoramiento, pues no saben cómo resolver los siguientes dilemas éticos (es importante que argumentes detalladamente tu respuesta. asi muesiros colegas sabrán exactamente lo que tienen que hacer.

1. Amanda es una psicóloga a la que ayer llegaron a su consulta los padres de una chica de 16 años que desean saber qué le está pasando a su hija, dado que su comportamiento se está volviendo cada vez mas alterado, no cuenta en casa las cosas que le suceden como hacia antes, y su rendimiento escolar está siendo inferior a lo que se espcra de ella A lo laro de lif

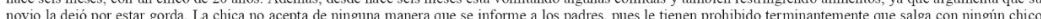
antes de los 18 anios, $y$ mos

2. Antonio trabaja como terapeuta en una clínica de enfermedades de transmisión sexual. La semana pesada tuvo la primera sesión con un paciente, quien le comunicé que habia dado recientemente positivo en la prueba de VIH, aunque no presenta sintomas. Antonio le explicó sobre la evolución de la enfermedad y las posibilidades de contagiar a otras personas. El paciente le confeso que, a pesar de tener pareja habia mantenido en los ult timos meses relaciones sexules de riesgo con otras personas. Antonio le habló sobre la necesidad de informar a su pareja sobre su diagnóstico, advirtiéndole del riesgo de transmitir el virus. Fl paciente se negó, argumentando que no queria perder a su pareja, y que no dijera nada a nadie, pues iba a cambiar y a dejar de mantener relaciones sexuales de riesgo. Antonio tiene dudas sobre este caso y nos pide ayuda para saber cómo actuar.

3. Raúl es un psicólogo al que acuden los padres de un chico de 15 años. Los padres lo han observado últimamente algo deprimido, apenas sale de su casa salvo para i al colegio o para visitar a los abuelos u otros familiares, no tiene amigos. El psicologo observa que el problema del chico es el excesivo control de los padres, quienes lo tratan como si fiera un nimo pequeno. Como principal objetivo del tratamiento, decide desarrollar un mayor sentido de autonomia. Durante varios meses todo va bien, el chaval ha comenzado a salir con amigos, a jugar al fútbol. Sin embargo, empieza a discutir con los padres por los horanos de salida y de estudio, y se mega a ir todos los fines de semana a visitar a los abuelos. Los padres se ponen en contacto con el psicólogo y le dicen que su hijo ahora es desobediente y estudia menos y que, por tanto, debe cambiar la forma de trabajar, ya que quieren que no esté depresivo, pero que siga siendo obediente. Raúl nos pide asesoramiento, pues no sabe cómo actuar en este momento

4. En estos momentos Beatriz está tratando a 10 pacientes en una clínica que desea abandonar para instalarse por su propia cuenta, pues no llega a final de mes con las condiciones económicas que le ofrecen. Reflexiona y dime cuatro medidas que Beatriz deberia tomar con respecto a la clinica y a sus pacientes, para actuar de una manera etica en este caso

5. Qué piensas sobre la amistad con un antiguo paciente? ¿Y sobre el hecho de mantener relaciones sexuales con un antiguo paciente?

6. Has empezado las prácticas del Grado en un Centro de mayores. Todo parece ir bien hasta que descubres que la mayoría de los días no ofrecen a sus usuarios la atención psicoterapéutica por la que han sido contratados y que, en su lugar, mantienen a los mayores en un salón durmiendo o viendo la televisión. Te dicen que el psicólogo que trabaja en el Centro proximamente se va a jubilar y que, si mantienes la boca cerrada y cumples las reglas, tú podrías ocupar su puesto una vez tengas el título. $i$ Qué harias?

Figura 3. Cuestionario inicial-final

\section{Aplicación del CIMA}

\section{Diario de sesiones}

A continuación, se expone el diario de las cuatro sesiones que componen la aplicación del presente CIMA. Se relatará de manera resumida el desarrollo acontecido relativo a las actividades diseñadas, se analizará brevemente mi actuación y mis sensaciones como docente, así como el clima de trabajo durante las distintas sesiones. Se comentarán también los avances y dificultades encontradas durante la aplicación del CIMA.

Al comienzo de la primera sesión, solicité a los alumnos que conformaran los grupos de trabajo, que serían de cuatro o cinco integrantes cada uno. Una vez formados, les informé que a partir de ese momento trabajaríamos con dilemas éticos. Les pregunté sobre sus ideas al respecto,

Ciclos de Mejora en el Aula (2020). Experiencias de Innovación Docente de la US Esta obra se distribuye con la licencia Creative Commons 
y si podían dar algún ejemplo de dilema y lo que esto implica para un profesional de la psicología. Esto llevó unos 15 minutos, pues al principio resultó más dificultoso hallar una adecuada participación activa de los estudiantes, por lo que llevó un poco más de tiempo de lo planeado que varios de los alumnos dieran su opinión. Igualmente, a la hora de pedir que uno de los grupos saliese voluntario, también costó la participación. Percibí que, para los dos casos vistos a lo largo de la sesión, introducir el debate exclusivo con el grupo moderador fomentó un gran clima de trabajo, al sentir los alumnos de este grupo que podían llevar la batuta. Por lo tanto, mi percepción fue que esta responsabilidad fue bien acogida entre los estudiantes, a pesar de ser algo novedoso para ellos. Precisamente el aumento en la participación fue el mayor cambio que noté frente a cursos pasados. El gran déficit otros años hacía referencia al planteamiento del debate en clase: el profesor presentaba un caso, el cual tenían que resolver los alumnos en grupos y exponerlo para toda la clase. El docente estimulaba, mientras tanto, un debate con el alumnado. Sin embargo, el resultado solía ser que solo tres o cuatro alumnos se animaban a debatir con el profesor, permaneciendo el resto de la clase en silencio o, como mucho, expresando un breve comentario. Este déficit fue en buena parte solucionado gracias a la innovación planteada con respecto al modelo metodológico. Por una parte, el grupo moderador se sentía protagonista después de debatir conmigo y obtener las consignas para moderar el debate posterior con el resto de los grupos. Por otra parte, todos los grupos participaron y expusieron su opinión, especialmente en el segundo caso de la sesión. En la primera mitad de la sesión noté, en líneas generales, al alumnado un poco perdido, pues no tenían apenas instrucciones sobre lo que tenían que hacer. Una vez visto el modelo de toma de decisiones tras el primero caso, se mostraron con mayor confianza para afrontar el segundo dilema, que además resultó de gran interés, suscitando un intercambio de Ciclos de Mejora en el Aula (2020). Experiencias de Innovación Docente de la US
Esta obra se distribuye con la licencia Creative Commons 
ideas entre los grupos más allá de la tarea propiamente dicho. Al tratar el caso sobre el aborto, se generó un ambiente de debate ideológico, en el cual algunas personas enfrentaron sus ideas favorables o contrarias a la temática del dilema. Esto provocó que finalmente tuviera que cortar el debate artificialmente, pues era la hora de terminar la clase, teniendo que presentar las conclusiones de la sesión de manera breve. La gestión del tiempo durante el debate tendré que cuidarla y mejorarla para el próximo curso, pero sin duda, mis sensaciones fueron más que positivas.

Con respecto a la segunda sesión, aproveché los primeros diez minutos para repasar y asentar las conclusiones extraídas de los casos vistos durante la sesión pasada, pues tuvieron que ser comentadas rápidamente por falta de tiempo. Recordé también a los alumnos el modelo de toma de decisiones para resolver los casos que veríamos durante la presente sesión. El primer caso generó un ambiente de enorme sensibilidad e indignación por parte del alumnado, por tratar un tema como el maltrato a pacientes internos. Tal y como ocurrió en la sesión anterior, el debate entre grupos llegó más allá de la mera resolución del caso, y surgió la inquietud por el hecho de hacer justicia sobre el mal ocasionado a los pacientes. Se explicó que la función del psicólogo en este caso no es juzgar, sino procurar que las acciones de abuso dejen de producirse. El debate entre los alumnos fue rico, tuve que intervenir para aclarar este tipo de cuestiones externas al caso, pero su resolución entró en el tiempo previamente establecido. El segundo caso también resultó de gran interés. Al ejercer de moderador en este caso el grupo de alumnos generalmente menos participativos, tuve que emplear más tiempo en el debate exclusivo con el grupo moderador, y tuve que intervenir un poco más en el debate con el resto de los grupos, pues les costaba hacerles las preguntas adecuadas a los compañeros. Aun así, llegamos entre todos a la resolución del caso, aunque de nuevo ajustado de tiempo, por lo que tuve que exponer las conclusiones brevemente. No obstante, mis sensaciones durante la clase fueron inmejorables.

Ciclos de Mejora en el Aula (2020). Experiencias de Innovación Docente de la US Esta obra se distribuye con la licencia Creative Commons 
En cuanto a la tercera sesión, comencé de nuevo repasando las conclusiones de la sesión anterior para afianzarlas. Esta vez lo hice un poco más rápido, para tener más margen para el adecuado desarrollo completo de la sesión. En esta ocasión no noté tanto interés por los casos como en las sesiones anterior, especialmente noté un menor entusiasmo con el segundo problema, el de las pseudoterapias, quizá porque hallaron su resolución demasiado fácil. En el primer dilema, del niño con problemas de lenguaje, sí se fomentó un mayor clima de trabajo e implicación. En cuanto a los grupos moderadores, ya costaba menor salir como voluntarios. En mi opinión, la autoeficacia de los alumnos iba incrementándose progresivamente, por lo que mostraban una mayor confianza. No obstante, en esta sesión, los grupos moderadores no estimularon el debate con el resto de grupos tanto como en ocasiones anteriores, dándoles las respuestas sin haberlas debatido y trabajado lo suficiente. Quizás ocurrió por este menor interés mencionado en las líneas anterior. Tuve por lo tanto que intervenir y lanzar algunas preguntas para hacer a los alumnos cuestionarse otras opciones de resolución del dilema, para que así el debate no fuera demasiado escaso. Tras exponer las conclusiones de la sesión y dar por finalizada la clase, mis sensaciones no fueron tan positivas como en las sesiones anteriores, y me fui de camino a casa pensando en que de cara al próximo curso tendré que dar una vuelta a la organización de esta sesión, reestructurando y modificando los casos para dotarlos de mayor interés y complejidad.

Por último, respecto a la cuarta sesión, recuperé las sesiones positivas perdidas en parte durante la sesión tercera. El primer caso, relativo al conflicto laboral, suscitó una adecuada implicación por parte de los alumnos, pues a raíz de algunas dudas que les surgieron derivadas de la temática del caso, percibí que lo vieron como una situación en la que probablemente se pueden ver inmersos en un futuro a corto/medio plazo. El debate generado fue enriquecedor, surgiendo un par de posturas que el grupo moderador supo confrontar y reconducir hacia la resolución 
recomendada para el caso. La gestión del tiempo en este caso fue adecuada, y afronté el segundo caso con la tranquilidad de que no habría que apresurarse para finalizar la clase adecuadamente. El segundo dilema supuso una grata sorpresa para mí, pues le tocó moderar el debate al grupo de alumnos menos participativos. Sin embargo, pude observar que su rol en la interacción con el resto de grupos creció significativamente, estimulando enormemente el debate y haciendo cuestionar a los compañeros sus propias ideas respecto a la resolución del caso. Además, integrando y haciendo propia la dinámica de clase, el mismo grupo moderador aportó espontáneamente las conclusiones del caso, sin yo haberlo solicitado previamente. Por lo que, tras la finalización del debate, únicamente tuve que ratificar lo comentado y contestar las dudas de algunos alumnos. Se generó un gran clima de trabajo, el alumnado se implicó activamente en el desarrollo de las actividades, y mis sensaciones fueron tremendamente positivas respecto al trabajo hecho.

\section{Evaluación del aprendizaje. Escaleras de aprendizaje}

Como se explicó anteriormente, para valorar el aprendizaje de los alumnos fue aplicado un cuestionario con seis preguntas, cada una de ellas enfocada en un problema clave. De los diecisiete alumnos del grupo, quince realizaron tanto el cuestionario inicial como el final. Para evaluar la evolución del aprendizaje, fueron analizadas y comparadas las respuestas de los estudiantes antes y después del CIMA. En las figuras 4 y 5 se muestran, a modo de ejemplo, las escaleras de aprendizaje obtenidas para las preguntas 1 (principio de confidencialidad) y 3 (límites de la patria potestad). Como puede observarse, se dio una notable mejoría en los modelos de respuesta de los alumnos. Así, para la pregunta 1 (figura 4), mientras que solo el $13.33 \%$ indicó en el cuestionario inicial que mantendrían la confidencialidad de la menor y negociarían con ella para que informe a sus padres sobre su problema (nivel 3), en

Ciclos de Mejora en el Aula (2020). Experiencias de Innovación Docente de la US Esta obra se distribuye con la licencia Creative Commons 
el cuestionario final el porcentaje de alumnos que apostó por la negociación ascendió hasta el 73.33\% (niveles 3 y 4). Igualmente, para la pregunta 3 (figura 5), mientras que solo el 13.33\% planteó inicialmente una negociación entre los progenitores y el menor, aportando propuestas de mediación entre las partes (nivel 3), en el cuestionario final este porcentaje subió hasta el $66.67 \%$ (niveles 3 y 4 ). Una similar tendencia se dio en las escaleras de aprendizaje del resto de preguntas, observándose una evolución significativa en los modelos de respuesta del grupo.

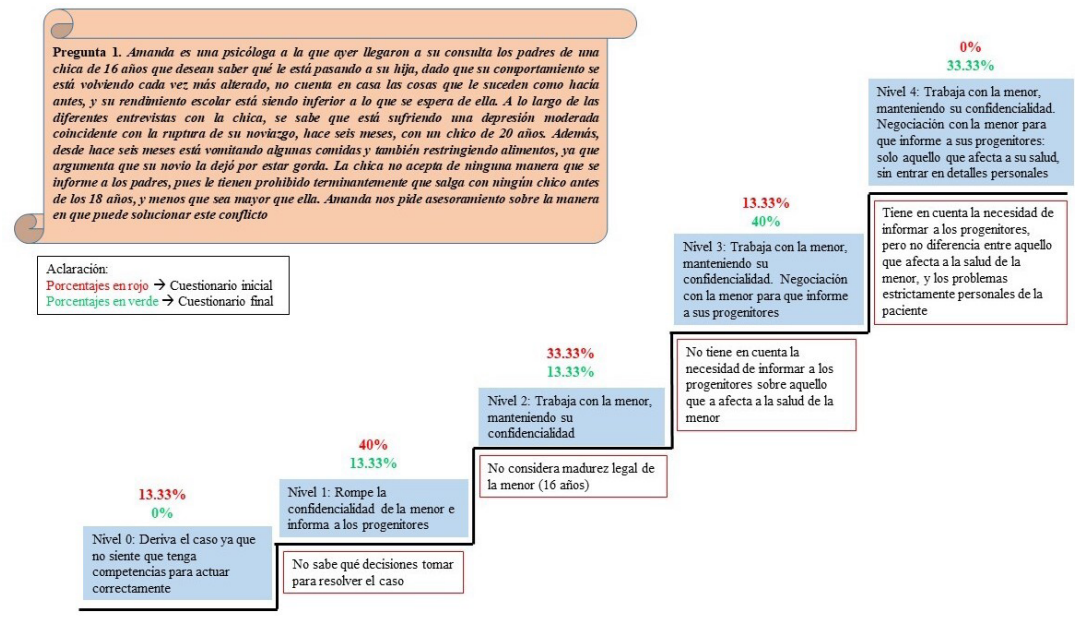

Figura 4. Escalera de aprendizaje para la primera pregunta del cuestionario

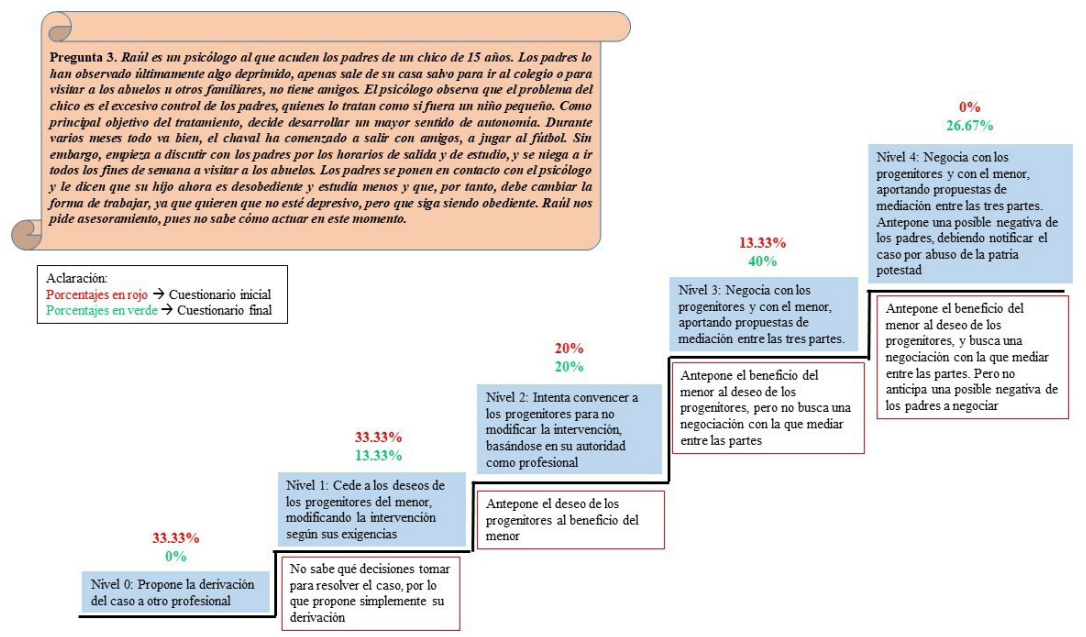

Figura 5. Escalera de aprendizaje para la tercera pregunta del cuestionario Ciclos de Mejora en el Aula (2020). Experiencias de Innovación Docente de la US
Esta obra se distribuye con la licencia Creative Commons 


\section{Evaluación del CIMA}

\section{Cuestiones a incorporar a la práctica docente habitual y cambios a introducir en el futuro}

En cuanto a la evaluación que hago de mi CIMA, el aspecto que más valoro es la notable evolución que han mostrado los alumnos en sus respuestas al cuestionario inicial-final, tal y como se ha descrito anteriormente, lo cual me lleva a interpretar que mis clases han facilitado el aprendizaje de los alumnos. La principal cuestión a mantener en el futuro es la transformación llevada a cabo en el modelo metodológico, pasando de un debate profesor-alumnos, en el cual el docente lleva el peso de la sesión, a un debate entre grupos de alumnos, donde ellos son los verdaderos protagonistas. Así, mientras que antes era yo el que llevaba la batuta de los debates, durante el CIMA la han llevado los distintos grupos moderadores. Un punto fuerte y enriquecedor de mi CIMA ha sido el debate exclusivo con el grupo moderador, pues los alumnos han expuesto sus ideas en un clima que les ha llevado a sentirse más seguros y confiados, aumentando su autoeficacia con respecto a la tarea realizada.

Considero que tanto el diseño del mapa de contenidos, como el modelo metodológico y la secuencia de actividades programadas han sido útiles para lograr un aprendizaje significativo en los alumnos, ya que, a partir de sus ideas previas, manifestadas tanto en el debate con el grupo moderador, como en el debate general, hemos construido el conocimiento, en este caso los pasos a seguir para resolver los dilemas éticos propuestos. Además, las preguntas seleccionadas para el cuestionario inicial-final creo que han sido un instrumento fiable para medir este aprendizaje, y coherentes con respecto al mapa de contenidos y al modelo metodológico: las cuestiones partieron de casos prácticos y no de definiciones o supuestos teóricos, y

Ciclos de Mejora en el Aula (2020). Experiencias de Innovación Docente de la US Esta obra se distribuye con la licencia Creative Commons 
se basaron en los problemas clave que han protagonizado el presente CIMA.

Como aspectos a revisar y mejorar en el futuro, destacaría la gestión del tiempo, pues en las dos primeras sesiones los casos abordados resultaron de gran interés, y el debate generado superó lo planeado previamente. Por lo tanto, debería reorientar la distribución de las sesiones y reservar más tiempo para el debate. Además, me gustaría en un futuro ser capaz de modificar sobre la marcha mi mapa de contenidos y secuencia de actividades en función de los resultados del cuestionario inicial, adaptando las clases a las ideas previas de los estudiantes.

Por otra parte, sería necesario realizar modificaciones y adaptaciones en los casos destinados a la tercera sesión, pues suscitaron menor interés y, en el caso de la intervención psicoterapéutica fraudulenta, su resolución resultó demasiado sencilla, por lo que parece que los alumnos permanecieron en su zona de confort. Asimismo, considero que en el futuro podría hacer un mayor seguimiento a aquellos alumnos más tímidos y menos participativos. Tanto en clase, procurando que tengan un mayor protagonismo, como fuera de clase, estimulando y reforzando positivamente su participación a través del correo electrónico.

Por último, las medidas de seguridad ante la situación de emergencia sanitaria han supuesto determinadas limitaciones para el desarrollo ideal de las clases. Algunos alumnos han manifestado problemas para seguir el hilo de los debates a causa de las mascarillas y los problemas de acústica que generan. Además, la distancia social en el aula ha entorpecido el debate dentro de cada grupo, y me ha impedido mostrarme tan cercano como hubiese deseado.

En definitiva, pienso que la aplicación de este CIMA ha sido un éxito en líneas generales, salvando las limitaciones y aspectos a mejorar. De esta manera, he podido comprobar que una docencia práctica y dinámica, en la que se tiene en cuenta las ideas previas de los alumnos, siendo

Ciclos de Mejora en el Aula (2020). Experiencias de Innovación Docente de la US Esta obra se distribuye con la licencia Creative Commons 
ellos los verdaderos protagonistas, facilita la implicación, motivación e interés, lo que redunda en una mayor satisfacción, tanto en los estudiantes como en el docente. Mi objetivo, en consecuencia, es incorporar y afianzar este modelo didáctico en mi rol como docente.

\section{Principios didácticos personales}

a) Los alumnos son los verdaderos protagonistas de la clase. Este sería mi principio didáctico de cabecera, al que más importancia doy, resume el ideal de lo que pretendo que sean mis clases durante mi carrera como docente. De este curso me llevo el compromiso de no caer en el modelo de enseñanza tradicional, aquel en el que el profesor habla y el alumno calla y escucha.

b) La interacción con el alumnado, y entre el alumnado, es una parte fundamental que nunca puede ser obviada. Debemos preocuparnos por conocer las ideas de nuestros alumnos sobre el contenido, y los alumnos deben percibir que los hacemos parte de la dinámica de la clase, que no son un mero decorado. Además, procurar que dialoguen y trabajen el contenido entre ellos. Fomentar el debate en clase es una excelente manera de crear un entorno para el aprendizaje crítico natural (Bain, 2004), generando a su vez un clima informal y distendido.

c) La sistematización de nuestro trabajo es el camino hacia una docencia rigurosa y exitosa. Al organizar previamente los contenidos en mapas, al tener claros los problemas clave, nos aseguramos de que estamos priorizando los contenidos más relevantes, siendo más probable que abordemos en clase todo aquello planeado previamente. Es mejor la calidad que la cantidad.

d) Aprendizaje significativo: debemos partir de las ideas previas de nuestros alumnos (Ausubel, 1976). Si no nos interesamos por conocer lo que el alumno conoce previamente sobre el contenido, nos podemos encontrar ante dos tipos de problemas en clase. Puede que estemos

Ciclos de Mejora en el Aula (2020). Experiencias de Innovación Docente de la US Esta obra se distribuye con la licencia Creative Commons 
enseñando algo que el alumno ya sabe, por lo que se quedará en su zona de confort. O puede que ocurra algo lamentablemente frecuente en la docencia universitaria: que partamos desde un nivel inalcanzable para el alumno, más allá de su zona de desarrollo próximo (Vygotsky, 1978). En consecuencia, debemos adaptarnos a las ideas previas de nuestros alumnos, y permitir que vayan progresivamente dando pasos fuera de su zona de confort, ampliando su zona de desarrollo próximo. Es aquí donde se produce el más valioso y enriquecedor aprendizaje.

e) La cercanía es vital para estimular un ambiente de aprendizaje en clase. Este es uno de los principales cambios que he percibido en mi trayectoria como docente. En mi primer año, debido a mi gran inseguridad y baja autoeficacia, me mantenía en una postura lejana a los alumnos, asegurándome de que no se perdiera esa jerarquía en clase que yo consideraba necesaria. Me situaba por lo tanto más en ese rol tradicional, transmisivo del conocimiento. Ahora mi confianza y autoeficacia ha aumentado, me permito mostrarme más cercano a mis alumnos, que me vean más como una figura orientadora del aprendizaje. Creo que durante las sesiones que han compuesto mi CIMA se ha generado un ambiente cercano y distendido con mis alumnos, lo cual ha facilitado mi satisfacción con el resultado de su aplicación.

f) Los trabajos en grupos reducidos sobre casos prácticos, o talleres conceptuales (Finkel, 2008), son una excelente manera de estimular y evaluar el aprendizaje. Asimismo, un sistema de evaluación basado en los portafolios o carpetas de aprendizaje, que permite al alumno hacer un seguimiento de su evolución a lo largo de la asignatura, y del proceso adoptado con respecto al aprendizaje de los contenidos, facilita una implicación activa y un aprendizaje significativo.

g) Las escaleras de aprendizaje y su utilidad dentro del marco de la evaluación (Porlán, 2017). Me quedo con el recurso de las escaleras, como método complementario 
al portafolio, para obtener una valoración global sobre el aprendizaje de los alumnos. Cuando comparamos ideas previas y finales estamos viendo, de un plumazo, cuantos pasos ha dado el alumno en su zona de desarrollo próximo, si el alumno realmente ha aprendido a resolver los problemas clave planteados. Supone también una gran oportunidad de cara al curso siguiente, pues unos resultados alejados de lo esperado serán sintomáticos de que algo ha fallado en nuestro modelo metodológico o en la secuencia de actividades programadas, pudiendo realizar las modificaciones necesarias. Debemos ser inconformistas y perfeccionistas en esta profesión.

Ciclos de Mejora en el Aula (2020). Experiencias de Innovación Docente de la US Esta obra se distribuye con la licencia Creative Commons 
Palabras clave: Ética y Deontología Profesional, Grado en Psicología, Docencia universitaria, Experimentación docente universitaria, Dilemas éticos.

Keywords: Professional Ethics and Deontology, Degree in Psychology, University Teaching, University Teaching Innovation, Ethical dilemmas.

\section{Referencias bibliográficas}

Ausubel, D. P. (1976). Psicología educativa. Un punto de vista cognoscitivo. México: Editorial Trillas.

Bain, K. (2004). Lo que hacen los mejores profesores universitarios. Valencia: Universitat de Valencia.

Canadian Psychological Association. (2017). Canadian Code of Ethics for Psychologists $4^{\text {th }} d$. Société Canadienne de Psychologie. Recuperado de http://cpa.ca/ docs/File/Ethics/CPA Code 2017 4thEd.pdf

Finkel, D. (2008). Dar clase con la boca cerrada. Valencia: Universitat de Valencia.

Porlán, R. (2017). Enseñanza universitaria. Cómo mejorarla. Madrid: Ediciones Morata.

Vygotsky, L. S. (1978). Mind in society: The development of higher psychological processes. Cambridge, MA: Harvard University Press.

Ciclos de Mejora en el Aula (2020). Experiencias de Innovación Docente de la US Esta obra se distribuye con la licencia Creative Commons 